\title{
Measuring plasma C4D to monitor immune complexes in lupus nephritis
}

\author{
Tineke Kraaij, ${ }^{1}$ Sara C. Nilsson, ${ }^{2}$ Cees van Kooten, ${ }^{1}$ Marcin Okrój, ${ }^{3}$ Anna M Blom, ${ }^{2}$ \\ YK Onno Teng ${ }^{1}$
}

To cite: Kraaij T, Nilsson SC, van Kooten $\mathrm{C}$, et al. Measuring plasma C4D to monitor immune complexes in lupus nephritis. Lupus Science \& Medicine 2019;6:e000326. doi:10.1136/ lupus-2019-000326

Received 12 February 2019 Revised 22 April 2019 Accepted 10 May 2019
Check for updates

(C) Author(s) (or their employer(s)) 2019. Re-use permitted under CC BY-NC. No commercial re-use. See rights and permissions. Published by BMJ.

${ }^{1}$ Department of Nephrology, Leiden University Medical Center, Leiden, The Netherlands ${ }^{2}$ Department of Translational Medicine, Section of Medical Protein Chemistry, Lund University, Malmö, Sweden ${ }^{3}$ Department of Medical Biotechnology, Intercollegiate Faculty of Biotechnology, University of Gdańsk and Medical University of Gdańsk, Gdańsk, Poland

Correspondence to

Tineke Kraaij; t.kraaij@lumc.nl

\section{ABSTRACT}

Objective Because currently available assays that measure circulating immune complexes (ICX) are suboptimal, a novel assay was recently developed measuring $\mathrm{C} 4 \mathrm{~d}$, a stable product of activation of the classical complement pathway. The present study aimed to establish the value of measuring plasma C4d levels in a longitudinal cohort of patients with severe refractory SLE who were treated with a combination therapy of rituximab with belimumab (RTX+BLM).

Methods Fifteen patients with SLE who were treated with $R T X+B L M$ in a phase $2 A$, open label study were included to sequentially measure plasma C4d levels and correlated to well-established markers of ICXformation, that is, autoantibodies against double-stranded (ds) DNA, autoantibodies against C1q and proteinuria. The performance of plasma C4d measurements, C4 measurements and the ratio of $\mathrm{C} 4 \mathrm{~d}$ over $\mathrm{C} 4(\mathrm{C} 4 \mathrm{~d}: \mathrm{C} 4)$ was evaluated.

Results After establishing that on RTX+BLM treatment kinetics of C4d levels was distinct from traditional C3 and $\mathrm{C} 4$ levels, we found strong correlation of $\mathrm{C} 4 \mathrm{~d}: \mathrm{C} 4$ with antidsDNA $(R=0.76, p<0.001)$ and anti-C1q $(R=0.65, p<0.001)$ autoantibody levels, which outperformed both stand-alone C4 and C4d levels. Additionally, changes in C4d:C4 over time correlated strongly with changes in proteinuria $(R=0.59, p<0.001)$ as well as anti-dsDNA ( $R=0.46$, $\mathrm{p}=0.003)$ and anti-C1q $(\mathrm{R}=0.47, \mathrm{p}=0.002)$.

Conclusion In patients with severe SLE, plasma C4d levels in relation to $\mathrm{C} 4$ levels is useful for longitudinal monitoring after RTX+BLM treatment to reflect amelioration of classical complement activation by ICX as well as proteinuria.

\section{INTRODUCTION}

Kidney involvement in SLE is typically defined by immunoglobulin deposition at the glomerular basement membrane (GBM) leading to membranoproliferative glomerulonephritis with the typical deposition of various complement components together with immunoglobulins referred to as 'fullhouse immunofluorescence'. The deposition of immunoglobulins in the kidney are caused by DNA or nucleosome containing immune complexes that can bind to the GBM and cause inflammation and damage through local as well as systemic complement activation $^{2}$ and as such complement activation products (such as C3d and C5b-9) can be detected in the urine of patients with SLE nephritis. ${ }^{3}$ The major cause of complement activation in SLE is thought to be the formation of immune complexes that activate complement via the classical pathway.

In clinical practice, immune complex (ICx)-mediated inflammation in patients with SLE is demonstrated by the consumption of complement components. Complement consumption is generally quantified with circulating C3 or C4 levels or functionally assessed by measuring complement classical pathway activity (CH50). Complement consumption has been found in approximately $75 \%$ of patients with SLE with focal proliferative glomerulonephritis and in $90 \%$ of patients with diffuse proliferative glomerulonephritis. ${ }^{4}$ However, a number of other factors not related to immune complexes or SLE disease activity per se may influence the degree of reduction of serum levels of complement components, including complement activation of the lectin or alternative pathway, ${ }^{5}$ the rate of complement production versus catabolism and the presence of autoantibodies directed against complement proteins, such as C1q. Therefore, several assays that (in-) directly measure circulating ICx have been developed with the intent to aid clinicians in their routine evaluations of ICx-mediated inflammation in patients with SLE. Since the currently available assays have thus far been insufficient to reliably and reproducibly detect immune complexes, ${ }^{6}$ a novel assay was recently developed measuring C4d, a stable product of activation of the classical complement pathway. ${ }^{7}$ We previously showed that plasma C4d levels is a functional readout for ICx-mediated classical pathway complement activation and associated with Systemic Lupus Erythematosus Disease Activity Index (SLEDAI), particularly nephritis and could forecast impending renal flares in patients with previous renal involvement. ${ }^{8}$ 
In the present study, we aimed to further establish the value of measuring plasma C4d levels in longitudinal cohort of patients with severe, refractory SLE who were treated with a combination therapy of rituximab (RTX) with belimumab (BLM) ${ }^{9}$ The cohort was selected because based on previous studies, the most severe patients with SLE were anticipated to have high C4d levels and a novel, synergetic B-cell targeted treatment was postulated to reduce, and even eradicate, ICx-forming autoantibodies. Therefore, optimising the clinical conditions to investigate sequentially measured plasma C4d levels in patients with SLE.

\section{METHODS}

\section{Study participants}

Patients with severe, refractory SLE who participated in a phase 2A, single-arm, proof-of-concept study investigating combination therapy of rituximab with belimumab (SynBioSe study, registered at ClinicalTrials.gov NCT02284984) were selected for this study. ${ }^{9}$ Patients were treated with $1000 \mathrm{mg}$ rituximab at weeks 0 and 2 and with monthly $10 \mathrm{mg} / \mathrm{kg}$ belimumab from week 4 and onwards. Only patients who completed 24 weeks of follow-up with serial serum sampling were selected. Details of the study participants, treatment and clinical efficacy were published elsewhere. ${ }^{9}$ All patients provided written informed consent.

\section{ELISA detecting soluble C4d}

Maxisorp 96-well plates (Nunc) were coated with rabbit anti-human C4d neoepitopes-specific antibody. ${ }^{7}$ After blocking with washing buffer $(50 \mathrm{mM}$ Tris-HCl, $0.15 \mathrm{M}$ $\mathrm{NaCl}, 0.1 \%$ Tween, $\mathrm{pH} 7.5$ ) supplemented with $3 \%$ fish gelatin (Norland Products), patient plasma as well as pooled plasma from healthy volunteers (lacking C4d) supplemented with Escherichia coli expressed C4d standard in serial dilutions, were diluted to $4 \%$ in phosphate-buffered saline $+0.02 \%$ Tween-20+0.02 $\mathrm{M} \mathrm{Na}_{2}$ EDTA and added to the plate for 1 hour incubation at $37^{\circ} \mathrm{C}$. Detection was achieved using mouse anti-human C4d antibody (Quidel, \#253) followed by horseradish peroxidase-conjugated goat antimouse secondary antibody (Dako, \#P0447). Plates were developed using OPD (Kem-En-Tec) as substrate and absorbance was measured at $490 \mathrm{~nm}$ using Cary50 microplate reader (Varian).

\section{Reference values}

In order to assess complement normalisation, clinical routine reference values were applied for C3 (normal $>0.9 \mathrm{~g} / \mathrm{L}$ ) and C4 (normal $>95 \mathrm{mg} / \mathrm{L}$ ). For C4d, reference values were based on previous published C4d levels and were categorised according to receiver operating characteristic curve analyses in a longitudinal cohort for lupus nephritis (normal $<1.1 \mathrm{mg} / \mathrm{L}$ ).

\section{Statistical analyses}

For patients' characteristics before and after treatment, descriptive analysis was used and median values reported

\begin{tabular}{ll}
\hline Table $1 \quad$ Patient characteristics & \\
\hline & $\mathbf{N}=15$ patients \\
\hline Age (years) & $31(19-51)$ \\
Sex (F/M) & $13 / 2$ \\
Disease duration (years) & $9(2-24)$ \\
Relapses (n) & $4(1-6)$ \\
Before treatment & \\
\hline SLEDAl & $18(6-29)$ \\
\hline Urine PCR (mg/mmol) & $107(11-852)$ \\
C4 (mg/L) & $79(21-260)$ \\
\hline C3 (g/L) & $0.6(0.3-1.3)$ \\
C4d (mg/L) & $2.05(0.98-6.40)$ \\
\hline Anti-dsDNA (IU/ml) & $144(18-505)$ \\
\hline Anti-C1q (U/ml) & $75.1(25.1-135)$ \\
After treatment (24 weeks) & \\
\hline SLEDAl & $2(0-13)^{\star}$ \\
\hline Urine PCR (mg/mmol) & $46(9-134)^{\star}$ \\
\hline C4 (mg/L) & $151(39-339)^{\star}$ \\
\hline C3 (g/L) & $1.0(0.5-1.4)^{\star}$ \\
\hline C4d (mg/L) & $1.25(0.17-3.62)^{\star}$ \\
\hline Anti-dsDNA (IU/ml) & $56.5(1-374)^{\star}$ \\
\hline Anti-C1q (U/ml) & $27.6(9.8-71.3)^{\star}$ \\
\hline
\end{tabular}

Depicted are median (range).

*Statistically significant $(p<0.05)$ difference from baseline. PCR, protein:creatinine ratio; SLEDAI, Systemic Lupus Erythematosus Disease Activity Index; dsDNA, double stranded DNA.

on the basis of the small cohort size. Non-parametric, two-tailed, paired samples Wilcoxon tests were used to compare treatment effects. To investigate the assay's performance, non-parametric Spearman's correlation coefficients were analysed.

\section{RESULTS}

Table 1 describes the patients' disease characteristics illustrating severe and refractory SLE and establishing that RTX+BLM indeed resulted in significant reductions in SLE relevant autoantibody levels. Anti-double stranded (ds) DNA levels declined from a median of $144 \mathrm{IU} / \mathrm{mL}$ (range 18-505) at baseline to 56.5 (1-374) at week $24(\mathrm{p}<0.001)$ and anti-C1q levels declined from $75.1 \mathrm{U} / \mathrm{mL}(25.1-135)$ to $27.6 \quad(9.8-71.3) \quad(\mathrm{p}<0.001)$. With respect to immune complex formation, the solidphase C1q binding test was positive in 5 of 15 patients. As a surrogate, serum levels of C3 and C4 were reduced at baseline and improved significantly on treatment from, respectively, median $0.6 \mathrm{~g} / \mathrm{L}(0.3-1.3)$ to $1.0 \mathrm{~g} / \mathrm{L}(0.5-$ 1.4) $(\mathrm{p}<0.001)$ and $80 \mathrm{mg} / \mathrm{L}(21-260)$ to 151 (39-339) $(\mathrm{p}=0.008)$. Plasma $\mathrm{C} 4 \mathrm{~d}$ levels were high at baseline with a median of $2.05 \mathrm{mg} / \mathrm{L}(0.98-6.40)$, in comparison to previously published C4d levels in patients with $\mathrm{SLE}^{8}$ and decreased significantly on treatment to $1.25 \mathrm{mg} / \mathrm{L}$ 
Table 2 Number (\%) of patients with complement activation before and normalisation after rituximab+belimumab

\begin{tabular}{rlllr}
\hline \multicolumn{2}{c}{ C4d } & C4 & \multicolumn{1}{l}{ C3 } & \multicolumn{1}{l}{ Total } \\
\hline \multicolumn{2}{r}{ Complement activation } \\
C4d & 14 & $9(64 \%)$ & $13(93 \%)$ & $14 / 15(93 \%)$ \\
C4 & $9(100 \%)$ & 9 & $8(89 \%)$ & $9 / 15(60 \%)$ \\
C3 & $13(100 \%)$ & $9(69 \%)$ & 13 & $13 / 15(87 \%)$ \\
\hline Complement normalisation & & \\
C4d & 6 & $6(100 \%)$ & $4(67 \%)$ & $6 / 14(43 \%)$ \\
C4 & $3(50 \%)$ & 6 & $4(67 \%)$ & $6 / 9(67 \%)$ \\
C3 & $3(50 \%)$ & $6(100 \%)$ & 6 & $6 / 13(46 \%)$ \\
\hline
\end{tabular}

SLEDAI, Systemic Lupus Erythematosus Disease Activity Index.

$(0.17-3.62) \quad(\mathrm{p}=0.01) \quad($ table 1$)$. We observed that at baseline, $\mathrm{C} 4 \mathrm{~d}$ levels were abnormal in 14 out of 15 patients $(93 \%)$ which was significantly more than C4 (abnormal in nine patients, equal to $60 \%$ ) and comparable to C3 (abnormal in 13 patients, equal to $87 \%$ ) (table 2). There was no significant difference for normalisation within 24 weeks of C4d (occurred in six patients $(43 \%)$ ), of C4 (in six patients (67\%)) and of C3 (in six patients (46\%)). Of interest, out of six patients whose C4d normalised, four patients had normalised C3 levels. Vice versa, out of six patients whose C3 normalised only half had normalised C4d levels suggestive of continued classical complement activation despite normalisation of $\mathrm{C} 3$ levels.

We next correlated C4d levels to C3 and C4 levels to establish that C4d levels reflected distinct aspects of complement activation. Indeed, we found no correlation between levels of $\mathrm{C} 4 \mathrm{~d}$ with either $\mathrm{C} 4$ levels $(\mathrm{R}=0.25$, $\mathrm{p}=0.38)$ nor $\mathrm{C} 3$ levels $(\mathrm{R}=-0.12, \mathrm{p}=0.66)$. Also, reduction of $\mathrm{C} 4 \mathrm{~d}$ levels did not correlate to increases in $\mathrm{C} 4(\mathrm{R}=0.02$, $\mathrm{p}=0.90)$ or $\mathrm{C} 3(\mathrm{R}=-0.20, \mathrm{p}=0.20)$.

Since levels of total C4 showed individual variation, we calculated the ratio of $\mathrm{C} 4 \mathrm{~d}$ activation product and total C4 (C4d:C4). The performance of these complement markers as a reflection of ICx-mediated inflammation was determined by comparison with other well-established markers of ICx-formation, that is, antibodies against dsDNA (anti-dsDNA) and C1q (anti-C1q) and proteinuria. We observed (figure 1A) the strongest correlations for $\mathrm{C} 4 \mathrm{~d}: \mathrm{C} 4$ with anti-dsDNA $(\mathrm{R}=0.76, \mathrm{p}<0.001)$ and anti-C1q $(\mathrm{R}=0.65, \mathrm{p}<0.001)$ autoantibodies which outperformed both $\mathrm{C} 4$ (respectively, $\mathrm{R}=-0.43, \mathrm{p}=0.001$ and $\mathrm{R}=-0.62, \mathrm{p}<0.001$ ) and $\mathrm{C} 4 \mathrm{~d}$ (respectively, $\mathrm{R}=0.67, \mathrm{p}<0.001$ and $\mathrm{R}=0.35, \mathrm{p}=0.01$ ). Of note, none of the complement markers correlated with proteinuria. Additionally, we investigated whether changes in complement markers accompanied changes in markers of ICx-formation (figure 1B). Again we found that changes in C4d:C4 over time had the strongest correlation with changes in antidsDNA ( $\mathrm{R}=0.46, \mathrm{p}=0.003)$, anti-C1q $(\mathrm{R}=0.47, \mathrm{p}=0.002)$ and proteinuria $(\mathrm{R}=0.59, \mathrm{p}<0.001)$. Likewise, changes in
C4d:C4 outperformed the stand-alone measurements of C4 and C4d.

\section{DISCUSSION}

The present study demonstrated that in patients with severe SLE measuring plasma C4d levels, and quantifying C4d:C4, was well correlated with current widely employed, surrogate measurements for ICx-formation (ie, antidsDNA, anti-C1q levels and proteinuria). In a cohort of patients with SLE with severe lupus nephritis, changes in C4d:C4 was strongly correlated with the renal outcome criterium of proteinuria and outperformed stand-alone C4d and C4 measurements. Also, on treatment, in some cases, C4d levels normalised, while C3 levels were still abnormal. Taken together, this study suggests that C4d quantification could be of added value to conventional complement measurements and warrants further validation for its use in the evaluation of immune complex-mediated diseases, including the wide spectrum of patients with mild to severe SLE, cryoglobulinemic glomerulonephritis or antibody-mediated humoral rejection.

Thus far, assays for measuring circulating immune complexes are insufficient because of the multiplicity of different techniques available for detecting immune complexes, differences in results when using different techniques, lack of reproducibility between the same techniques performed in different laboratories, and lack of studies demonstrating clear clinical predictive value of immune complex measurements. ${ }^{6}$ As such, the lack of detectable circulating immune complexes does not exclude the presence of immune complex-mediated diseases, including involvement of complement activation. ${ }^{10}$ Therefore, there is a clinical need for the development of more specific biomarkers of classical pathway complement activation. ${ }^{3}{ }^{11}$ The assay for quantifying plasma C4d levels employs neoantigen-specific antibodies to nascent cleavage epitope found only in $\mathrm{C} 4 \mathrm{~d}$ but not its parent molecules such as $\mathrm{C} 4$ and C4b. ${ }^{7}$ Of note, patients with a genetic variation at the $\mathrm{C} 4$ null alleles can impact the rate of production and catabolism of $\mathrm{C} 4$ and $\mathrm{C} 4 \mathrm{~d}$. To correct this phenomenon, the ratio of C4d:C4 can be calculated or serial measurements within the same patient can be performed, as exemplified in this study.

Previously, in patients with SLE, the deposition of C4d on reticulocytes and platelets was shown to associate with SLEDAI $^{12}$ and was suggested as useful biomarker for monitoring disease activity. ${ }^{13}$ A prospective pilot study showed that levels of C4d on circulating cells correlated with C4d staining in lupus nephritis biopsies. ${ }^{14}$ An assay panel combining anti-dsDNA, ANA, anti-mutated citrullinated vimentin, erythrocyte C4d and B-cell C4d was shown to be sensitive and specific for the diagnosis of SLE. ${ }^{15}$ The present study is the first to add to this body of evidence that C4d levels in relation to C4 levels is a useful marker for longitudinal monitoring in the setting of treatment reflecting amelioration of classical complement activation by immune complexes. Future studies 
A
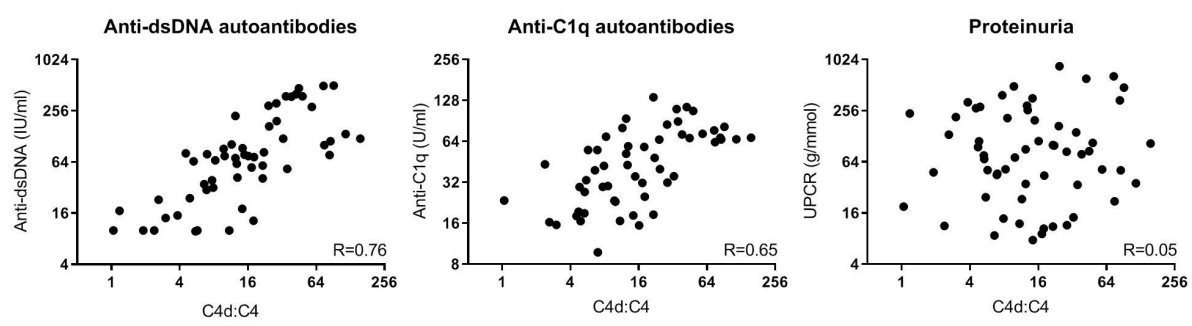

\begin{tabular}{l|cc|cc|cc}
\hline & \multicolumn{2}{|c|}{ C4 } & \multicolumn{2}{c|}{ C4d } & \multicolumn{2}{c}{ C4d:C4 } \\
& $\mathbf{R}$ & $\mathbf{P}$ & $\mathbf{R}$ & $\mathbf{P}$ & $\mathbf{R}$ & $\mathbf{P}$ \\
\hline Anti-dsDNA & -0.43 & 0.001 & 0.67 & $<0.001$ & 0.76 & $<0.001$ \\
Anti-C1q & -0.62 & $<0.001$ & 0.35 & 0.01 & 0.65 & $<0.001$ \\
Proteinuria (UPCR) & 0.17 & 0.19 & 0.19 & 0.15 & 0.05 & 0.73 \\
\hline
\end{tabular}

B
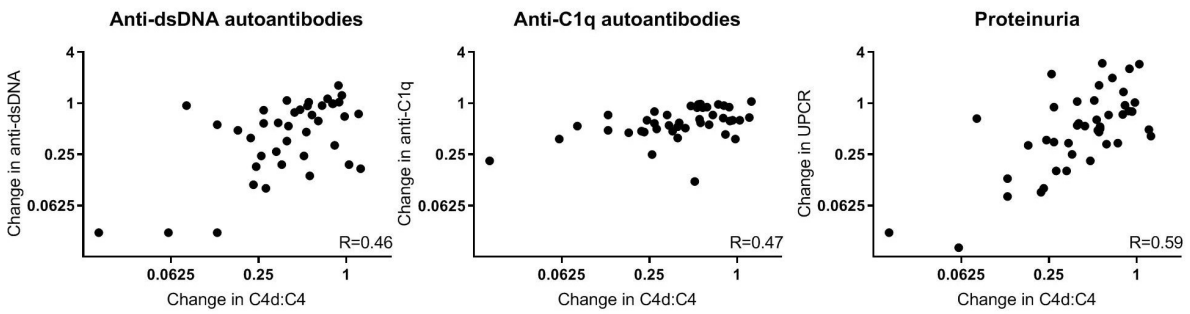

\begin{tabular}{l|cc|cc|cc}
\hline Change in & \multicolumn{2}{|c|}{ C4 } & \multicolumn{2}{c|}{ C4d } & \multicolumn{2}{c}{ C4d:C4 } \\
& $\mathbf{R}$ & $\mathbf{P}$ & $\mathbf{R}$ & $\mathbf{P}$ & $\mathbf{R}$ & $\mathbf{P}$ \\
\hline Anti-dsDNA & -0.21 & 0.18 & 0.22 & 0.17 & 0.46 & 0.003 \\
Anti-C1q & -0.08 & 0.61 & $\mathbf{0 . 4 9}$ & $<0.001$ & 0.47 & 0.002 \\
Proteinuria (UPCR) & -0.53 & $<0.001$ & 0.30 & 0.048 & $\mathbf{0 . 5 9}$ & $<0.001$ \\
\hline
\end{tabular}

Figure 1 Significant association of plasma C4d levels with traditional, surrogate markers for immune complex formation. (A). Levels of $\mathrm{C} 4 \mathrm{~d}$ and $\mathrm{C} 4$ were measured simultaneously from which the $\mathrm{C} 4 \mathrm{~d}: \mathrm{C} 4$ ratio was derived and correlated to circulating levels of autoantibodies directed against double stranded (ds) DNA and C1q and proteinuria (measured by the urine protein:creatinine ratio (UPCR)). The correlation plots of the ratio of $\mathrm{C} 4 \mathrm{~d}: \mathrm{C} 4$ illustrate strong correlation with levels of anti-dsDNA and anti-C1q autoantibodies ( $\mathrm{R}=$ Spearman's correlation coefficient). The table reports the correlation coefficients separately for C4d, C4 and C4d:C4 where strong correlations $(R \geq 0.5)$ are depicted in dark grey filled boxes; moderate correlation $(0.3<R<0.5)$ are depicted in light grey filled boxes; no or weak correlations $(R \leq 0.3)$ are depicted in non-filled boxes. $P$ values were considered significant when below 0.05. (B). Change in levels of C4d, C4 and C4d:C4 in comparison to baseline levels were calculated. The relative changes of $\mathrm{C} 4 \mathrm{~d}, \mathrm{C} 4$ and $\mathrm{C} 4 \mathrm{~d}: \mathrm{C} 4$ were correlated to the relative changes in circulating levels of autoantibodies directed against dsDNA, C1q and proteinuria. Depicted graphs illustrate the correlation of changes in C4d:C4 with changes in anti-dsDNA ( $R=0.46 ; p=0.003)$ and anti-C1q $(R=0.47 ; p=0.002)$ autoantibody levels and, most relevantly, changes in proteinuria $(R=0.59 ; p<0.001)$. In the table, correlation coefficients are reported separately for changes in C4d, C4 and C4d:C4.

will need to elucidate how clinical decision-making can benefit from routine $\mathrm{C} 4 \mathrm{~d}: \mathrm{C} 4$ measurements.

Contributors TK, SN, CVK, AMB and YKOT contributed to the ideas for the article. TK, CVK, AMB and YKOT analysed the data, participated in the interpretation of the data and wrote and revised the manuscript. AMB and MO were involved in developing the assay for C4d measurement. All authors approved of the final version.

Funding The study was funded by the Swedish Research Council (2016-01142), King Gustav V's 80th Anniversary Foundation, Swedish Rheumatism Association, and grants for clinical research (ALF and from the Skåne University Hospital). Dr Kraaij and Dr Teng's work is supported by the Dutch Kidney Foundation (KJPB12.028 and 170KG04), Clinical Fellowship from the Netherlands Organization for Scientific Research (90713460). Marcin Okrój's work is supported by the National Science Centre Poland, grant no. 2014/14/E/NZ6/00182. GlaxoSmithKline (GSK) provided belimumab and an unrestricted grant for the clinical study described in this manuscript.

Competing interests $\mathrm{AMB}$ and $\mathrm{MO}$ are named as inventors in a patent application including claims to use of $\mathrm{C} 4 \mathrm{~d}$ as biomarker.
Patient consent for publication Not required.

Ethics approval The study was approved by the LUMC medical ethics committee (Commissie Medische Ethiek LUMC, P14.065).

Provenance and peer review Not commissioned; externally peer reviewed. Data sharing statement No additional data are available.

Open access This is an open access article distributed in accordance with the Creative Commons Attribution Non Commercial (CC BY-NC 4.0) license, which permits others to distribute, remix, adapt, build upon this work non-commercially, and license their derivative works on different terms, provided the original work is properly cited, appropriate credit is given, any changes made indicated, and the use is non-commercial. See: http://creativecommons.org/licenses/by-nc/4.0/.

\section{REFERENCES}

1. Rijnink EC, Teng YKO, Wilhelmus S, et al. Clinical and histopathologic characteristics associated with renal outcomes in lupus nephritis. Clin J Am Soc Nephrol 2017;12:734-43.

2. van der Vlag J, Berden JHM. Lupus nephritis: role of antinucleosome autoantibodies. Semin Nephrol 2011;31:376-89. 
3. Manzi S, Rairie JE, Carpenter AB, et al. Sensitivity and specificity of plasma and urine complement split products as indicators of lupus disease activity. Arthritis Rheum 1996;39:1178-88.

4. Valentijn $\mathrm{RM}$, van Overhagen $\mathrm{H}$, Hazevoet $\mathrm{HM}$, et al. The value of complement and immune complex determinations in monitoring disease activity in patients with systemic lupus erythematosus. Arthritis Rheum 1985;28:904-13.

5. Chen M, Daha MR, Kallenberg CGM. The complement system in systemic autoimmune disease. J Autoimmun 2010;34:J276-J286.

6. Wener $\mathrm{MH}$. Tests for circulating immune complexes. Methods $\mathrm{Mol}$ Biol 2014;1134:47-57.

7. Blom AM, Österborg A, Mollnes TE, et al. Antibodies reactive to cleaved sites in complement proteins enable highly specific measurement of soluble markers of complement activation. Mol Immunol 2015;66:164-70.

8. Martin M, Smoląg KI, Björk A, et al. Plasma C4d as marker for lupus nephritis in systemic lupus erythematosus. Arthritis Res Ther 2017;19.

9. Kraaij T, Kamerling SWA, de Rooij ENM, et al. The NET-effect of combining rituximab with belimumab in severe systemic lupus erythematosus. J Autoimmun 2018;91:45-54.

10. Use and abuse of laboratory tests in clinical immunology: critical considerations of eight widely used diagnostic procedures. Report of a joint IUIS/WHO meeting on assessment of tests used in clinical immunology. Clin Immunol Immunopathol 1982;24:122-38.

11. Buyon JP, Tamerius J, Belmont HM, et al. Assessment of disease activity and impending flare in patients with systemic lupus erythematosus. Comparison of the use of complement split products and conventional measurements of complement. Arthritis Rheum 1992;35:1028-37.

12. Liu C-C, Manzi S, Kao AH, et al. Reticulocytes bearing C4d as biomarkers of disease activity for systemic lupus erythematosus. Arthritis Rheum 2005;52:3087-99.

13. Kao AH, Navratil JS, Ruffing MJ, et al. Erythrocyte C3d and C4d for monitoring disease activity in systemic lupus erythematosus. Arthritis Rheum 2010;62:837-44.

14. Batal I, Liang K, Bastacky S, et al. Prospective assessment of C4d deposits on circulating cells and renal tissues in lupus nephritis: a pilot study. Lupus 2012;21:13-26.

15. Kalunian KC, Chatham WW, Massarotti EM, et al. Measurement of cell-bound complement activation products enhances diagnostic performance in systemic lupus erythematosus. Arthritis Rheum 2012;64:4040-7. 